\title{
Neutrophil to lymphocyte ratio is associated with proximal/middle segment of the LAD lesions in patients with ST segment elevation infarction
}

\author{
OZLEM ARICAN OZLUK ${ }^{1}$, MUSTAFA YILMAZI, DURSUN TOPAL ${ }^{2}$, ERHAN TENEKECIOGLU ${ }^{l}$, \\ TEZCAN PEKER ${ }^{l}$, SELCUK KANAT ${ }^{l}$, KEMAL KARAAGAC ${ }^{l}$, FAHRIYE VATANSEVER ${ }^{l}$, \\ UTKU PARLAK
}

'Department of Cardiology, Bursa Ihtisas Training and Research Hospital, Bursa, Turkey

${ }^{2}$ Department of Cardiology, Sevket Yilmaz Training and Research Hospital, Bursa, Turkey

\begin{abstract}
Introduction: Neutrophil to lymphocyte ratio (NLR) was revaled to have a close relation with atherosclerotic cardiovascular disease. The relationship between NLR and culprit plaque localization has never been studied.

Aim of the study: To evaluate the association between NLR and unstable plaque localization of left anterior descending artery (LAD) in anterior miyocardial infarction patients.

Material and methods: Patients admitted to our hospital with acute anterior STEMI were included. Fifhy-eight patients who have single-vessel disease at LAD and their hematological parameters were analyzed retrospectively. Proximal segment of LAD lesions were groupped as Group I and mid segment of the LAD lesion groupped as Group II. The groups were compared according to their NLR and other parameters.

Results: Between group I $(n=41$, mean age $52.5 \pm 12.7)$ and group $I I(n=17$, mean ages 52.0 $\pm 10.8)$; NLR, were significantly higher in group I compared to the group II (6.9 $\pm 5.6 \mathrm{vs.} 3.3 \pm 2.0$, $p=0.01)$. In group I, left ventricular ejection fraction $(L V E F)$ was significantly lower $(p=0.02)$. In correlation analyzes, NLR was positively correlated with $C K M B(r=0.32, p=0.01)$ and negatively correlated with LVEF ( $r=-0.28, p=0.03)$.

Conclusions: The present study demonstrated that anterior myocardial infarction patients with high $N L R$ had a greater possibility having proximal culprit lesion on the LAD. Therefore NLR can be used as a useful tool to culprit plaque localization in patients with acute miyocardial infarction patients.
\end{abstract}

Key words: coronary artery disease, neutrophil to lymphocyte ratio, acute coronary syndrome.

(Cent Eur J Immunol 2016; 41 (4): 386-391)

\section{Introduction}

Cardiovascular disease including ST-segment elevation myocardial infarction (STEMI), is a significant disease with its morbidity and mortality. STEMI characterized by an acute occlusion of blood flow to the myocardial territory supplied by the infarct-related artery (IRA). Atherosclerosis is the main cause of coronary artery disease (CAD) [1,2]. Some factors have been reported to be independently related to STEMI mortality $[3,4]$. Localization of culprit lesion is one of the significant factor can determine the clinical outcome in STEMI.

It is known that inflammation plays a crucial role in atherosclerotic coronary artery disease $[5,6]$. In recent years, neutrophil to lymphocyte ratio (NLR) derived from the peripheral blood accept as a new prognostic predictor of atherosclerotic coronary artery disease and acute coronary syndromes [3, 4, 7-15]. It was shown that NLR was a poor prognostic marker in acute coronary syndromes. Recently, it was also reported that NLR was an independent predictor of mortality in patients with STEMI $[11,13,15]$.

However, to our knowledge, there are no data about the relationship between NLR and localization of coronary artery culprit lesion yet. Therefore, the aim of this study was to investigate the usefulness of neutrophil to lymphocyte ratio, in predicting the localization of the culprit coronary artery lesion in anterior myocardial infarction.

Correspondence: Dr. Ozlem Arican Ozluk, Kardiyoloji Klinigi, Bursa Yuksek Ihtisas Egitim ve Arastirma Hastanesi, Yildirim Bursa,

Turkey, tel. +902243605050, fax +902243605055, e-mail: oarican@yahoo.com

Submitted: 27.08.2015; Accepted: 23.11.2015 


\section{Material and methods}

In this study, retrospective analysis of 137 patients was performed who underwent primary percutaneous intervention at the cardiology service of our hospital between 2012 and 2014 for acute anterior myocardial infarction. Fifty-eight patients who have only proximal or mid culprit lesion on LAD vessel complied with the inclusion criteria were included in this study. The exclusion criteria included the following; patients who had received fibrinolytic therapy, angiographically any significant stenosis in the right or circumflex coronary artery together with an LAD artery lesion, distal LAD lesions, patients with a history of previous coronary artery disease, trauma, surgery, neoplasm or infectious disease in the last 30 days prior to hospitalization as well as current use of immunosuppressant (including corticosteroids) were excluded from the study

Acute anterior STEMI was identified by new left bundle branch block or new ST elevation at the J point in two contiguous leads with the cut-points: $0.1 \mathrm{mV}$ in anterior leads other than leads V2-V3 where the following cut points apply: $\geq 0.2 \mathrm{mV}$ in men and $\geq 0.15 \mathrm{mV}$ in women and typical chest pain lasting 20 minutes or longer [16]. In all patients, the culprit lesion was shown by coronary angiography for confirming the diagnosis of acute anterior myocardial infarction. Hyperlipidemia was diagnosed as low-density cholesterol (LDL) was greater than $100 \mathrm{mg} / \mathrm{dl}$ or lipid-lowering medication was used. Blood pressure of all patients was measured. Systolic blood pressure (SBP) equal or greater than $140 \mathrm{mmHg}$ and/or diastolic blood pressure equal or greater than $90 \mathrm{mmHg}$ or under control by using blood pressure-lowering medications were defined as hypertension. Patients with a fasting glucose level equal or greater than $126 \mathrm{mg} / \mathrm{dl}$ on two separate occasions or using oral antidiabetic drugs or insulin were defined as diabetes mellitus.

Proximal segment of LAD lesions were groupped as Group I and mid segment of the LAD lesion groupped as Group II. The groups were compared according to their neutrophil to lymphocyte ratios (NLR) and other parameters.

\section{Analysis of blood samples}

Complete blood count and basic biochemical parameters were evaluated retrospectively from blood samples obtained by antecubital vein puncture upon 1 hour after admission to the hospital. Peripheral venous blood samples were drawn into K3 EDTA containing biochemistry tubes. Hematologic indices are evaluated from complete blood count analysis performed using a Coulter LH 780 Hematology Analyzer (Beckman Coulter Ireland Inc, Mervue, Galway, Ireland). Other biochemical measurements were determined by standard laboratory methods.

\section{Echocardiography}

All patients underwent a complete transthoracic echocardiography (TTE) and Doppler study using multiple views in left lateral decubitus position. Echocardiographic examination was performed within 24 hours of admission. TTE study was performed using a $3.5 \mathrm{MHz}$ transducer on a GE Vivid 7 Pro device. Echocardiographic measurements were made according to the criteria recommended by American Society of Echocardiography [17]. Left ventricular end-diastolic volume (LVEDV), left ventricular end- systolic volume (LVESV), and ejection fraction (LVEF) were measured from the apical 4-chamber view using the modified Simpson method.

\section{Angiography}

All patients underwent coronary angiography to determine the infarct-related artery and revascularization. Determination of infarct-related artery was accomplished by observing a thrombosed critical stenosis of LAD in certain locations. The proximal to and including first major septal branch is classified as the proximal LAD, while the segment just below the first major septal branch is the mid LAD. The distal segment of the LAD is the terminal third of the artery. A narrowing of $\geq 70 \%$ in other vessels were defined as additional vessel disease.

\section{Statistical analysis}

Statistical analyses were performed using the SPSS software version 16.0 (SPSS Inc., Chicago, Illinois, USA). The study population was divided into two groups based on the localization of the LAD lesions. Descriptive statistics are given in the mean \pm standard deviation while the categorical variables were expressed as number and percentage (\%). In order to investigate the distribution of data, Kolmogorov Smirnov test was used. Normally distributed variables compared across groups by means of student $t$ test. The nonparametric variables compared using the Mann-Whitney $U$ test. Spearman's test was used for correlation analysis between neutrophil to lymphocyte ratio and other parameters. Receiver operating characteristic (ROC) curves for NLR values were plotted to determine the optimal cut-off values for individual parameters in order to predict the level of culprit lesion on the left anterior descending artery and to establish the optimal cut-off points for use in clinical decision-making. A $p$ value less than 0.05 was considered statistically significant for all the statistical assessments.

\section{Results}

The study population was classified according to their localization of the LAD lesions. Forty-one patients with proximal LAD lesion were defined as Group I, seventeen patients with mid LAD lesion were defined as Group II. The demographic, biochemical, echocardiographic data of the two groups were compared (Tables 1,2 ). 
The two groups did not significantly differ with respect to the major cardiovascular risk factors such as hypertension, diabetes mellitus, smoking, and heredity. Both groups were similar with regard to mean age and gender distribution.

Comparison of the groups with respect to the laboratory data revealed that Group I had significantly higher cardiac injury marker; CK-MB $(p<0.05)$. Neutrophil to lymphocyte ratio (NLR) was significantly higher in the group I compared to group II ( $p=0.01)$ Lymphocyte count was significantly lower in group I $(p<0.05)$. No significant differences were noted between the other routine biochemical data of both groups.

In group I, left ventricular ejection fraction was significantly lower $(p<0.05)$, left ventricular end-systolic volume were significantly higher $(p<0.05)$ (Table 3$)$.

In correlation analyzes, NLR was positively correlated with CKMB $(r=0.32, p=0.01)$, troponin $\mathrm{I}(r=0.25$, $p=0.02)$ and LVESV $(r=0.28, p=0.03)$. Also There was a negative correlation between NLR and LVEF $(r=-0,28$, $p=0.03$ ) (Table 4). Regarding the diagnostic accuracy of the NLR, we calculated the composite score for sensitivity and specificity (i.e. the area under the curve (AUC), as determined by the ROC curve analysis for the prediction of the level of culprit lesion on the left anterior descending artery by the NLR. Overall, the AUC was 0.712 (95\% CI: 0.581-0.843;

Table 1. Demographic and biochemical characteristics of study groups

\begin{tabular}{|c|c|c|c|}
\hline Parameters & $\begin{array}{l}\text { Group I } \\
(n=41)\end{array}$ & $\begin{array}{c}\text { Group II } \\
(n=17)\end{array}$ & $\begin{array}{c}P \\
\text { values }\end{array}$ \\
\hline Age (years) & $52.5 \pm 12.7$ & $52.0 \pm 10.8$ & 0.87 \\
\hline Male, $n(\%)$ & $37(90.2 \%)$ & $13(76.5 \%)$ & 0.16 \\
\hline \multicolumn{4}{|l|}{ Coronary risk factors } \\
\hline Smoking, $n(\%)$ & $23(56 \%)$ & $11(65 \%)$ & 0.54 \\
\hline Hypertension, $n(\%)$ & $7(17 \%)$ & $3(17 \%)$ & 0.95 \\
\hline Diabetes mellitus, $n(\%)$ & $2(4.9 \%)$ & $2(11.8 \%)$ & 0.34 \\
\hline Family History, $n(\%)$ & $2(4.9 \%)$ & $2(11.8 \%)$ & 0.34 \\
\hline \multicolumn{4}{|l|}{ Biochemical parameters } \\
\hline Glucose (mg/dl) & $129 \pm 36.1$ & $121 \pm 34$ & 0.47 \\
\hline Urea (mg/dl) & $15.0 \pm 4.1$ & $14.6 \pm 3.6$ & 0.74 \\
\hline Creatinin (mg/dl) & $0.81 \pm 0.2$ & $0.75 \pm 0.2$ & 0.28 \\
\hline HDL-cholesterol (mg/dl) & $38.1 \pm 11.2$ & $39.4 \pm 10.7$ & 0.74 \\
\hline LDL-cholesterol (mg/d) & $114 \pm 29$ & $117.3 \pm 44.4$ & 0.76 \\
\hline Triglyserid (mg/dl) & $122 \pm 85$ & $126.9 \pm 94$ & 0.72 \\
\hline CK-MB (U/l) & $248 \pm 200$ & $152 \pm 140$ & 0.04 \\
\hline Troponin I (ng/ml) & $186.9 \pm 118$ & $103.1 \pm 100$ & 0.02 \\
\hline \multicolumn{4}{|c|}{$\begin{array}{l}\text { LDL - low density lipoprotein; } H D L-\text { high density lipoprotein; } C K-M B- \\
\text { creatine kinase myocardial band; } W B C-\text { white blood cell; } N L R-\text { neutrophi } \\
\text { to lymphocyte ratio } \\
\text { Data are presented as means } \pm S D\end{array}$} \\
\hline
\end{tabular}

$p=0.012$ ). Using cut-off value of 4.7 , the sensitivity and specificity of the NLR were $40 \%$ and $55 \%$, respectively (Fig. 1).

\section{Discussion}

In the present study, we found that NLR at admission was significantly increased in patients with anterior myocardial infarction who have proximal left anterior descending (LAD) lesion. In addition we also showed that LVEF levels were significantly lower and cardiac enzyme were significantly higher in patients with proximal left anterior descending lesion.

ST elevation myocardial infarction is common cause of mortality and morbidity in developed countries. Myocardial ischemia results in life-threatening complications including electrical instability, left ventricular remodeling and valvular dysfunction. Patients with STEMI should undergo risk stratification soon after presentation to predict those who are at high risk for short and long term adverse outcomes. Hypotension, Killip class II-IV, heart rate $>100$ bpm, elevated serum creatinine, cardiac arrest during pre-

Table 2. Hemogram parameters of study groups

\begin{tabular}{|c|c|c|c|}
\hline Parameters & $\begin{array}{l}\text { Group I } \\
(n=41)\end{array}$ & $\begin{array}{l}\text { Group II } \\
(n=17)\end{array}$ & $\begin{array}{c}P \\
\text { values }\end{array}$ \\
\hline $\begin{array}{l}\text { WBC count } \\
\left(10^{3} / \mathrm{mm}^{3}\right)\end{array}$ & $12.65(9.3-15.9)$ & $11.95(9.1-14.7)$ & 0.45 \\
\hline $\begin{array}{l}\text { Neutrophil } \\
\text { count } \\
\left(10^{3} / \mathrm{mm}^{3}\right)\end{array}$ & $10(3.1-17.7)$ & $7.6(4.3-12.9)$ & 0.15 \\
\hline $\begin{array}{l}\text { Lymphocyte } \\
\text { count } \\
\left(10^{3} / \mathrm{mm}^{3}\right)\end{array}$ & $1.5(0.7-14.0)$ & $2.1(1.4-6.7)$ & 0.03 \\
\hline $\begin{array}{l}\text { Eosinophils } \\
\left(10^{3} / \mathrm{mm}^{3}\right)\end{array}$ & $0.10(0.01-0.30)$ & $0.11(0.01-0.22)$ & 0.12 \\
\hline $\begin{array}{l}\text { Monocytes } \\
\left(10^{3} / \mathrm{mm}^{3}\right)\end{array}$ & $0.60(0.02-0.85)$ & $0.80(0.58-1.02)$ & 0.65 \\
\hline NLR & $6.9(1.1-24.3)$ & $3.3(0.9-6.7)$ & 0.01 \\
\hline $\begin{array}{l}\text { Platelet count } \\
\left(10^{3} / \mathrm{mm}^{3}\right)\end{array}$ & $\begin{array}{c}231.02 \\
(161-291.1)\end{array}$ & $243.88(161-308)$ & 0.19 \\
\hline
\end{tabular}

NLR - neutrophil to lymphocyte ratio

Data are presented as median with minimum and maximum values.

Table 3. Echocardiographic parameters in study groups

\begin{tabular}{cccc}
\hline Parameters & $\begin{array}{c}\text { Group I } \\
(\boldsymbol{n}=\mathbf{4 1})\end{array}$ & $\begin{array}{c}\text { Group II } \\
(\boldsymbol{n}=\mathbf{1 7})\end{array}$ & $\begin{array}{c}\boldsymbol{P} \\
\text { values }\end{array}$ \\
\hline LVEDV $(\mathrm{ml})$ & $98.8 \pm 27.3$ & $83.4 \pm 14.1$ & 0.06 \\
\hline LVESV $(\mathrm{ml})$ & $53.1 \pm 21.6$ & $38.9 \pm 9.8$ & $\mathbf{0 . 0 2}$ \\
\hline LVEF $(\%)$ & $37.8 \pm 9.2$ & $46.3 \pm 8.2$ & 0.02 \\
\hline LVEDV - left ventricular end-diastolic volume; LVESV - left ventricular end-
\end{tabular}

systolic volume; $L V E F$ - left ventricular ejection fraction 
Table 4. Correlations between the neutrophil to lymphocyte ratio and the patient variables

\begin{tabular}{lcc}
\hline Parameters & $\boldsymbol{r}$ values & $\boldsymbol{P}$ values \\
\hline Age & 0.42 & 0.75 \\
\hline Creatinin & 0.89 & 0.89 \\
\hline CK-MB & 0.32 & 0.01 \\
\hline Troponin I & 0.25 & 0.02 \\
\hline LVEF & -0.28 & 0.03 \\
\hline LVEDV & 0.24 & 0.11 \\
\hline LVESV & 0.28 & 0.03 \\
\hline $\begin{array}{l}\text { CK-MB - creatine kinase myocardial band; LVEF - left ventricular ejection } \\
\text { fraction; LVEDV - left ventricular end-diastolic volume; } \text { LVESV - left } \\
\text { ventricular end-systolic volume }\end{array}$ &
\end{tabular}

sentation, higher baseline white blood cell (WBC) count are among of the risk factors. Atherosclerotic stenosis of the proximal segment of the left anterior descending coronary artery (LAD) constitute a special subgroup of coronary artery disease (CAD), given the high-risk profile [18-21]. Because of the myocardium at risk is relatively large. This affects its prognosis. This patients require specific and early intervention. Previous studies showed that proximal culprit LAD lesions have been associated with malignant ventricular arrhythmias and higher incidence of adverse outcomes in ST elevation acute myocardial infarction $[22,23]$. By estimating the the level of LAD coronary lesion in STEMI patients can decrease complication rates; because more agresive treatment options can be applied earlier. Unfortunately, at this point noninvasive methods give limited data about the location of the LAD lesions. Even electrocardiography, which is most used test, may remain insufficient to predict the level of coronary obstruction in anterior myocardial infarction [24, 25].

In this context, neutrophil to lymphocyte ratio derived from routine complete blood count test is an distinctive, inexpensive, easily available inflammatory indicator. It is known that inflammation plays an integral role in atherosclerosis [5, 6] and NLR is associated with enhanced inflammatory response [26]. Previous studies reported that white blood cell subtypes especially the neutrophil-to-lymphocyte ratio has been shown that to be related to a proinflammatory state imposing worse clinical outcomes in patients with cardiovascular disease $[8,10]$ The relationship between the atherosclerotic cardiac disease and NLR is shown in many studies [3, 4, 8-15].

We demonstrated that patients with high NLR had more proximal LAD lesions. There is no study in the literature investigating the relationship between neutrophil to lymphocyte ratio and the level of culprit lesion in anterior myocardial infarction patients. Therefore, present study gains importance as it is the first study in this field.

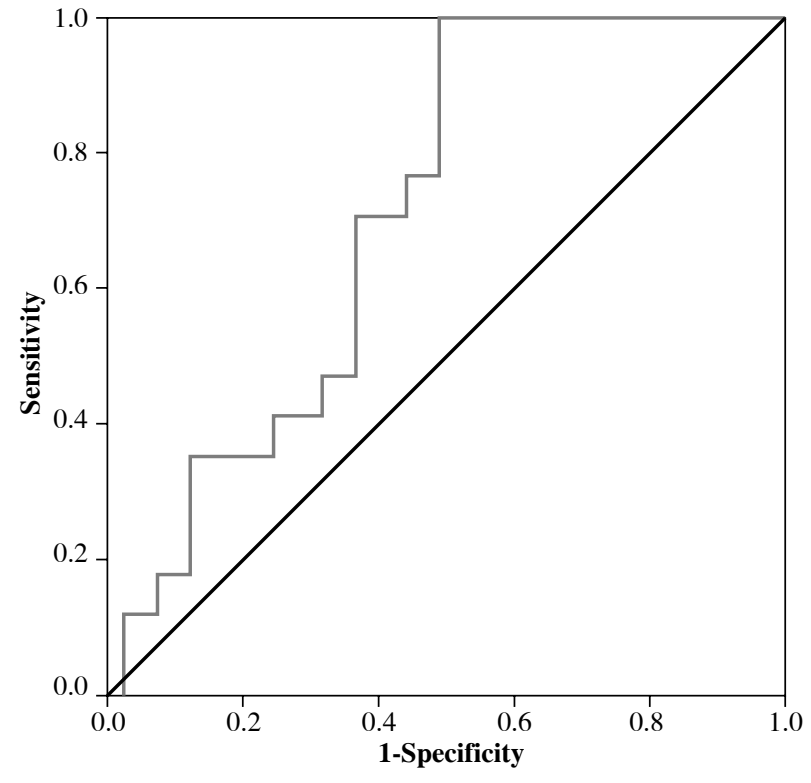

Fig. 1. Receiver operating characteristic (ROC) curve analysis for prediction of the level of culprit lesion by (NLR). The area under the curve AUC) is 0.712 (95\% CI: 0.581 $0.843 ; p=0.012$ ) for NLR

Choi et al. reported that coronary artery disease patients with high NLR had more vulnerable plaque components than those with low NLR [27]. It is known that vulnerable plaque in STEMI patients tended to within the proximal segments of these vessels [28, 29]. Recent study by Fowler $e$ al. showed that NLR is related to the severity of coronary artery disease [30]. In the present study, we suggest that patients with high NLR have a greater possibility having proximal vulnerable plaque which lead to anterior myocardial infarction. In accordance with the literature, in the present study, patients with proximal lesion have lower left ventricular ejection fraction and higher cardiac injury marker: CK-MB and troponin I. In our population, most of the patients had proximal lesion, similar to epidemiologic studies results. Although our study indicated that proximal LAD lesions have higher NLR; this ratio is also associated with infarct size. In the our study group, the degree of myocardial dysfunction (based on the LVEF) and the level of cardiac enzymes (CK MB and troponin I) in the blood were associated with NLR values. Proximal lesion is one of the determinants of infarct size but it is difficult to make the distinction which one is more affected on the NLR value. Hence, patients who have very limited anatomic coronary lesions were enrolled in the study. We excluded distal LAD lesions and the another coronary lesions. In this study we would like to draw attention to single vessel proximal lesions anterior myocardial infarction have higher NLR values. These simple marker, NLR, 
is easily available parameter and inexpensive in routine clinical measurement. It may be useful during admissions to hospital for detecting the high risk profile patients in anterior myocardial infarction.

There are some limitations of our study. The basic limitations of our study are the small sample size, lack of long-term follow-up data and a retrospective study. Other limitations may include the enrollment of acute coronary syndrome patients only anterior myocardial infarction. Therefore, there is a need for future studies with larger sample size underwent primary PCI that will explore the relationship between neutrophil to lymphocyte ratio levels and the level of culprit lesions in myocardial infarction and evaluate the long-term outcomes of that relationship.

\section{Conclusions}

In conclusion, the present study demonstrated that anterior myocardial infarction patients with high NLR had a greater possibility having proximal culprit lesion on the LAD. Therefore NLR can be used as a useful tool to detect not only significant atherosclerosis but also culprit plaque localization in patients with acute STEMI patients.

\section{The authors declare no conflict of interest.}

\section{References}

1. Crouse JR 3rd, Grobbee DE, O'Leary DH, et al. (2004): Measuring Effects on intima media Thickness: an Evaluation Of Rosuvastatin in subclinical atherosclerosis - the rationale and methodology of the METEOR study. Cardiovasc Drugs Ther 18: 231-238.

2. Rauch U, Osende JI, Fuster V, et al. (2001): Thrombus formation on atherosclerotic plaques: pathogenesis and clinical consequences. Ann Intern Med 134: 224-238.

3. Tamhane UU, Aneja S, Montgomery D, et al. (2008): Association between admission neutrophil to lymphocyte ratio and outcomes in patients with acute coronary syndrome. Am J Cardiol 102: 653-657.

4. Núńez J, Núńez E, Bodí V, et al. (2008): Usefulness of the neutrophil to lymphocyte ratio in predicting long-term mortality in ST segment elevation myocardial infarction. Am J Cardiol 101: 747-752.

5. Hansson GK (2005): Inflammation, atherosclerosis, and coronary artery disease. N Engl J Med 352: 1685-1695.

6. Weissberg PL (2000): Atherogenesis: current understanding of the causes of atheroma. Heart 83: 247-252.

7. Horne BD, Anderson JL, John JM, et al. (2005): Which white blood cell subtypes predict increased cardiovascular risk? J Am Coll Cardiol 45: 1638-1643.

8. Duffy BK, Gurm HS, Rajagopal V, et al. (2006): Usefulness of an elevated neutrophil to lymphocyte ratio in predicting long-term mortality after percutaneous coronary intervention. Am J Cardiol 97: 993-996.

9. Núńez J, Núńez E, Bodí V, et al. Usefulness of the neutrophil to lymphocyte ratio in predicting long-term mortality in ST segment elevation myocardial infarction. Am J Cardiol 2008; 101: 747-752.

10. Muhammed Suliman MA, Bahnacy Juma AA, Ali Almadhani AA, et al. (2010): Predictive value of neutrophil to lymphocyte ratio in outcomes of patients with acute coronary syndrome. Arch Med Res 41: 618-622.

11. Cho KH, Jeong MH, Ahmed K, et al. (2011): Value of early risk stratification using hemoglobin level and neutrophil-to-lymphocyte ratio in patients with ST-elevation myocardial infarction undergoing primary percutaneous coronary intervention. Am J Cardiol 107: 849-856.

12. Arbel Y, Finkelstein A, Halkin A, et al. (2012): Neutrophil/ lymphocyte ratio is related to the severity of coronary artery disease and clinical outcome in patients undergoing angiography. Atherosclerosis 225: 456-460.

13. Park JJ, Jang HJ, Oh IY, et al. (2013): Prognostic value of neutrophil to lymphocyte ratio in patients presenting with ST-elevation myocardial infarction undergoing primary percutaneous coronary intervention. Am J Cardiol 111: 636-642.

14. Yilmaz M, Tenekecioglu E, Arslan B, et al. (2015): White blood cell subtypes and neutrophil-lymphocyte ratio in prediction of coronary thrombus formation in non-ST-segment elevated acute coronary syndrome. Clin Appl Thromb Hemost 21: 446-452.

15. Acet H, Ertaş F, Bilik MZ, et al. (2015): The relationship between neutrophil to lymphocyte ratio, platelet to lymphocyte ratio and thrombolysis in myocardial infarction risk score in patients with ST elevation acute myocardial infarction before primary coronary intervention. Postep Kardiol Inter 11: 126-135.

16. Thygesen K, Alpert JS, Jaffe AS, et al. (2012): Third universal definition of myocardial infarction. J Am Coll Cardiol 60: 1581-1598.

17. Lang RM, Bierig M, Devereux RB, et al. (2005): Recommendations for chamber quantification: a report from the American Society of Echocardiography's Guidelines and Standards Committee and the Chamber Quantification Writing Group, developed in conjunction with the European Association of Echocardiography, a branch of the European Society of Cardiology. J Am Soc Echocardiography 12: 1440-1463.

18. Valencia J, Borders P, Berenguer A, et al. (2002): Long term follow-up of patients with proximal left anterior descending coronary artery stenosis treated with stent. Rev Esp Cardiol 55: 607-615.

19. Carrizo S, Peinado RP, Sanchez-Recalde A, et al. (2015): Clinical and angiographic characteristics of patients with acute coronary syndrome associated with sudden cardiac death. Hellenic J Cardiol 56: 136-141.

20. Califf RM, Tomabechi Y, Lee KL, et al. (1983): Outcome in one-vessel coronary artery disease. Circulation 67: 283-290.

21. Klein LW, Weintraub WS, Agarwal JB, et al. (1986): Prognostic significance of severe narrowing of the proximal portion of the left anterior descending coronary artery. Am J Cardiol 58: 42-46.

22. Arnar DO, Bullinga JR, Martins JB (1997): Role of the Purkinje system in spontaneous ventricular tachycardia during acute ischemia in a canine model. Circulation 96: 2421-2429.

23. Kwofie MA, Chaudhary AK, Martins JB (2011): Association among intracardiac T-wave alternans, ischemia, and spontaneous ventricular arrhythmias after coronary artery occlusion in a canine model. Transl Res 158: 265-272. 
24. Colaco R, Reay P, Beckett C, et al. (2000): False positive ECG reports of anterior myocardial infarction in women. J Electrocardiol 33: 239-24.

25. Figueras J (2002): Non precordial leads and the level of coronary obstruction in acute anterior myocardial infarction: something clinically useful or only redundant information? Rev Esp Cardiol 55: 1015-1017.

26. Imtiaz F, Shafique K, Mirza SS, et al. (2012): Neutrophil lymphocyte ratio as a measure of systemic inflammation in prevalent chronic diseases in Asian population. Int Arch Med 5: 1-6.

27. Choi YH, Hong YJ, Ahn Y, et al. (2014): Relationship between Neutrophil-to-Lymphocyte Ratio and Plaque Components in Patients with Coronary Artery Disease: Virtual Histology Intravascular Ultrasound Analysis. J Korean Med Sci 29: 950-956.

28. Gibson CM, Kirtane AJ, Murphy SA, et al. (2003): Distance from the coronary ostium to the culprit lesion in acute ST-elevation myocardial infarction and its implications regarding the potential prevention of proximal plaque rupture. J Thromb Thrombolysis 15: 189-196.

29. Wang JC, Normand SL, Mauri L, et al. (2004): Coronary artery spatial distribution of acute myocardial infarction occlusions. Circulation 110: 278-284.

30. Fowler AJ, Agha RA (2013): Neutrophil/lymphocyte ratio is related to the severity of coronary artery disease and clinical outcome in patients undergoing angiography: the growing versatility of NLR. Atherosclerosis 228: 44-45. 